\title{
Evaluation of female Aedes aegypti proteome via LC-ESI- MS/MS using two protein extraction methods
}

\author{
Abubakar Shettima ${ }^{1,2}$, Intan Haslina Ishak ${ }^{3,4}$, Syahirah Hanisah Abdul Rais ${ }^{1}$, Hadura Abu Hasan ${ }^{3,4}$, Nurulhasanah \\ Othman ${ }^{\text {Corresp. } 1}$ \\ 1 Institute for Research in Molecular Medicine (INFORMM), Universiti Sains Malaysia, Gelugor, Pulau Pinang, Malaysia \\ 2 Department of Microbiology, Faculty of Science, University of Maiduguri, Maiduguri, Borno State, Nigeria \\ 3 School of Biological Sciences, Universiti Sains Malaysia, Gelugor, Pulau Pinang, Malaysia \\ ${ }^{4}$ Vector Control Research Unit, School of Biological Sciences, Universiti Sains Malaysia, Gelugor, Pulau Pinang, Malaysia \\ Corresponding Author: Nurulhasanah Othman \\ Email address: nurulhasanah@usm.my
}

Background. Proteomic analyses have broadened the horizons of vector control measures by identifying proteins associated with different biological and physiological processes and give further insight into the mosquitoes' biology, mechanism of insecticide resistance and pathogens-mosquitoes interaction. Female Ae. aegypti ingests human blood to acquire the requisite nutrients to make eggs. During blood ingestion, female mosquitoes transmit different pathogens. Therefore, this study aimed to determine the best protein extraction method for mass spectrometry analysis which will allow a better proteome profiling for female mosquitoes. Methods. In this present study, two protein extractions methods were performed to analyze female Ae. aegypti proteome, via TCA acetone precipitation extraction method and a commercial protein extraction reagent CytoBuster $^{\mathrm{TM}}$. Then, protein identification was performed by LC-ESI-MS/MS and followed by functional protein annotation analysis. Results. CytoBuster ${ }^{\top \mathrm{M}}$ reagent gave the highest protein yield with a mean of $475.90 \mu \mathrm{g}$ compared to TCA acetone precipitation extraction showed $283.15 \mu \mathrm{g}$ mean of protein. LC-ESI-MS/MS identified 1290 and 890 proteins from CytoBuster $^{\mathrm{TM}}$ reagent and TCA acetone precipitation, respectively. When comparing the protein class categories in both methods, there were three additional categories for proteins identified using CytoBuster ${ }^{T M}$ reagent. The proteins were related to scaffold/adaptor protein (PC00226), protein binding activity modulator (PC00095) and

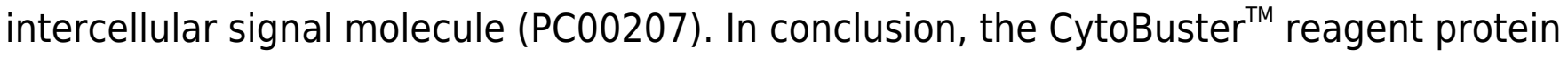
extraction reagent showed a better performance for the extraction of proteins in term of the protein yield, proteome coverage and extraction speed. 


\section{Evaluation of female Aedes aegypti proteome via LC-}

2 ESI-MS/MS using two protein extraction methods

3

4

5

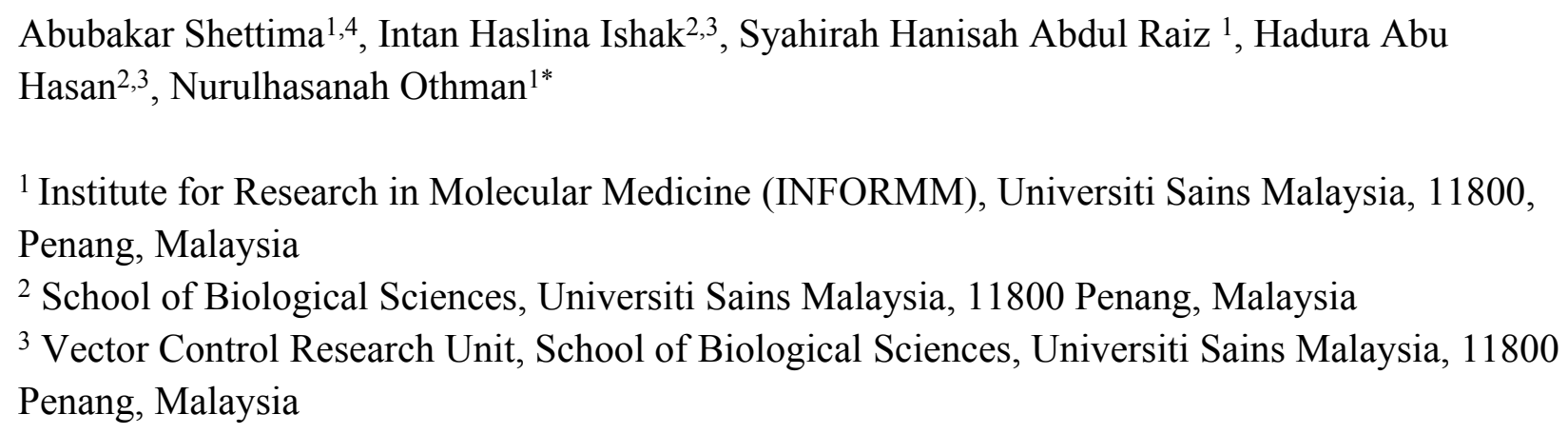

\section{Abstract} Background.

Proteomic analyses have broadened the horizons of vector control measures by identifying proteins associated with different biological and physiological processes and give further insight 
39 into the mosquitoes' biology, mechanism of insecticide resistance and pathogens-mosquitoes

40 interaction. Female Ae. aegypti ingests human blood to acquire the requisite nutrients to make

41 eggs. During blood ingestion, female mosquitoes transmit different pathogens. Therefore, this

42 study aimed to determine the best protein extraction method for mass spectrometry analysis

43 which will allow a better proteome profiling for female mosquitoes.

44 Methods.

45 In this present study, two protein extractions methods were performed to analyze female $\mathrm{Ae}$.

46 aegypti proteome, via TCA acetone precipitation extraction method and a commercial protein

47 extraction reagent CytoBuster ${ }^{\mathrm{TM}}$. Then, protein identification was performed by LC-ESI-MS/MS

48 and followed by functional protein annotation analysis.

49 Results.

50 CytoBuster ${ }^{\mathrm{TM}}$ reagent gave the highest protein yield with a mean of $475.90 \mu \mathrm{g}$ compared to TCA

51 acetone precipitation extraction showed $283.15 \mu \mathrm{g}$ mean of protein. LC-ESI-MS/MS identified

521290 and 890 proteins from CytoBuster ${ }^{\mathrm{TM}}$ reagent and TCA acetone precipitation, respectively.

53 When comparing the protein class categories in both methods, there were three additional

54 categories for proteins identified using CytoBuster ${ }^{\mathrm{TM}}$ reagent. The proteins were related to

55 scaffold/adaptor protein (PC00226), protein binding activity modulator (PC00095) and

56 intercellular signal molecule (PC00207). In conclusion, the CytoBuster ${ }^{\mathrm{TM}}$ reagent protein

57 extraction reagent showed a better performance for the extraction of proteins in term of the

58 protein yield, proteome coverage and extraction speed.

59 Keyword: Aedes aegypti, protein extraction methods, protein identification, LC-ESI-MS/MS

60 


\section{Introduction}

63 Proteomics is a fast-developing research field. Scientist experimented continuously to profile and

64 catalogue insect proteome, including mosquitoes at different tissues and organelle and in varying

65 physiological states (Shashank and Haritha, 2014). Furthermore, their interactions with viruses,

66 parasites and toxins were also studied (Shashank and Haritha, 2014).

67

68 In general, mosquito proteomic analyses have revealed features of haemolymph proteins, midgut 69 peritrophic matrix proteins, mosquito-head proteins during different feeding (sugar or blood)

70 (Shashank and Haritha, 2014; Whiten et al., 2018). Proteomics using LC-MS/MS (Liquid

71 Chromatography-Mass Spectrometry) also made possible the elucidation of host-virus

72 interactions (Shashank and Haritha, 2014). Female Ae. aegypti ingest human blood to acquire the

73 nutrients necessary to produce eggs. During blood ingestion, female mosquitoes may transmit

74 different pathogens, including viruses such as dengue and yellow fever to their respective host.

75 Several studies have investigated differential protein expressions in mosquitoes to elucidate 76 protein regulations during various physiological conditions (Popova-Butler and Dean, 2009;

77 Djegbe et al., 2011; Cancino-Rodezno et al., 2012; Wang et al., 2015; Whiten et al., 2018; Mano

78 et al., 2019). Protein functional classification analysis also reveals their biological processes,

79 molecular function, and cellular components as well as phylogenic and ancestral strings by the

80 functional annotation and detailed bioinformatics analysis (Shashank and Haritha, 2014).

81

82 It is critical to determine which extraction protocol produce high protein yield and achieve more

83 protein hits from protein identification by LC-ESI-MS/MS analysis. Therefore, this study

84 compared two extraction methods for protein identification analysis in female Ae. aegypti, by

85 following a TCA acetone precipitation method by Wang et al., and a commercial protein 
86

87

88

89

90

91

92

93

94 95

9

extraction reagent CytoBuster ${ }^{\mathrm{TM}}$ (Sigma, Germany) to assess the performance of the extraction method for LC-ESI-MS/MS analysis which will allow a better proteome profiling for female mosquitoes.

\section{Materials \& Methods}

\section{Mosquito samples}

Ae. aegypti eggs papers were obtained from the Vector Control Research Unit (VCRU), Universiti Sains Malaysia (USM), and reared at the Insectary of the School of Biological

4 Sciences, Universiti Sains Malaysia, at a constant room temperature of approximately $28^{\circ} \mathrm{C}$ and $75 \%$ relative humidity. Eggs were submerged in water and applied a larvae food composed of grounded dog biscuit, beef liver, powdered milk and yeast in a ratio of 2:1:1:1. Pupae from the larvae were transferred into half-full disposable cups and placed in a cage net for adult mosquitoes to emerge. Adult mosquitoes were fed with $10 \%$ sucrose solution before harvesting and 3-5 days old female mosquitoes were harvested for the protein extraction at the Institute for Research in Molecular Medicine (INFORMM), USM.

\section{Protein extraction}

Three biological replicates comprised of 20 adult female mosquitoes in each replicate, were analyzed for each extraction method. For the TCA acetone precipitation method (Wang et al., 2015), female adult Ae. aegypti were washed three times with distilled deionized water to remove food particles and molted skin before homogenization at 50rpm for 5-10 mins in a mini bead beater using $0.5 \mathrm{~mm}$ zirconia beads in $10 \%$ TCA cold acetone and 10mM DTT, and incubated overnight at $-20^{\circ} \mathrm{C}$. Then centrifuged at $4^{\circ} \mathrm{C}, 15000 \times \mathrm{g}$ for 5 mins and the pellets resuspended in lysis buffer (7M urea, 2M Thiourea, 4\% CHAPS) containing 1mM PMSF, 2mM 
109 EDTA and 10mM DTT. The mixture was sonicated for $1 \mathrm{~min}$, with 10-sec pulse and $10 \mathrm{sec}$ stop,

110 and centrifuged at $4^{\circ} \mathrm{C}, 15000 \times \mathrm{g}$ for $5 \mathrm{mins}$. The supernatant was reduced and alkylated with

111 10mM DTT and 55mM IAA, respectively. Then the sample was precipitated with chilled

112 acetone (1:4) followed by incubation at $-20^{\circ} \mathrm{C}$ overnight. Then, the precipitant was resuspended

113 in 10mM Tris HCL, then sonicated for $1 \mathrm{~min}$, with 10 -sec pulse and $10 \mathrm{sec}$ stop, and centrifuged

114 at $4^{\circ} \mathrm{C}, 15000 \times \mathrm{g}$ for 5 mins. The supernatant was collected and used for subsequent analysis.

115

116 Using CytoBuster ${ }^{\mathrm{TM}}$ extraction reagent, a total of 20 female adult Ae. aegypti mosquitoes in each

117 replicate were homogenized in $600 \mu \mathrm{L}$ of the reagent in a mini bead beater using $0.5 \mathrm{~mm}$ zirconia

118 beads at $50 \mathrm{rpm}$ for $5 \mathrm{mins}$ at room temperature, and the pellets were then centrifuged at $16000 \mathrm{x}$

$119 g$ at $4^{\circ} \mathrm{C}$ for 5 mins. The supernatant was transferred into a new tube and concentrated $6 \mathrm{X}$ using

120 spin columns with 10,000 molecular weight cut-off (MWCO) (GE Healthcare) at $4000 \times g$, at

$1214^{\circ} \mathrm{C}$ for 30 mins. After that, the $1 \mathrm{x}$ final concentration of protease inhibitor (Sigma, Germany)

122 was added to the protein extract from both methods and kept at $-20^{\circ} \mathrm{C}$.

\section{Protein separation}

124 After protein quantification was performed by reducing agent and detergent compatible

$125\left(\mathrm{RCDC}^{\mathrm{TM}}\right)$ Protein Assay (Biorad, USA), a total of $20 \mu \mathrm{g}$ of protein from each biological

126 replicate and method was heated to $99^{\circ} \mathrm{C}$ for $5 \mathrm{mins}$ in SDS loading buffer. The protein samples

127 were loaded onto $10 \%$ SDS PAGE gel and run at 200-V for 20 mins until they became stacked

128 on the top of the separating gel. Then, the gel was stained in RAMA stain and incubated for $1 \mathrm{~h}$

129 on a rocker. The staining solution was removed and then washed with distilled water for 3-5

130 times until bright protein bands were visible. 


\section{In-gel digestion}

133 A protein band comprised of the whole protein mixture from each replicate and method, as

134 mentioned before, was excised from the gel into small pieces and put into a $1.5 \mathrm{ml}$ centrifuge

135 tube. Then $200 \mu \mathrm{L}$ of a destaining solution made from $80 \mathrm{mg}$ ammonium bicarbonate with $20 \mathrm{~mL}$

$136 \mathrm{ACN}$ and $20 \mathrm{~mL}$ ultrapure water was added to the gel pieces and incubated at $37^{\circ} \mathrm{C}$ for 30 mins

137 with shaking at $300 \mathrm{rpm}$. The solution mixture was discarded and the process was repeated three

138 times to destain the gels completely. After that, $200 \mu \mathrm{L}$ fresh reducing buffer (10mM DTT in

$139100 \mathrm{mM}$ ammonium bicarbonate) was added to cover the gels and incubated at $60^{\circ} \mathrm{C}$ for 30 mins.

140 The gels were allowed to cool, and the buffer was removed. Then, $200 \mu \mathrm{L}$ fresh alkylating buffer

141 (55mM IAA in 100mM AMBIC) was added and incubated in the dark for 60 mins, and the

142 solution was discarded. The gel pieces were washed with a destaining solution and kept at $37^{\circ} \mathrm{C}$

143 for 15 mins, shaking at 300rpm, and then the destaining solution was discarded. After reducing

144 and alkylating, $50 \mu \mathrm{L}$ ACN was added to shrink the gel and incubated for 15 mins at room

145 temperature. The ACN was discarded, and the gel was allowed to air dry for 5-10 mins. Then, 30

$146 \mu \mathrm{L}$ of $12.5 \mathrm{ng} / \mathrm{uL}$ trypsin (Promega, USA) was added to the gel pieces, and the tube was covered

147 with a parafilm and incubated at $37^{\circ} \mathrm{C}$ overnight shaking at $300 \mathrm{r}$ pm. The digestion mixture was

148 transferred to a clean tube and labelled appropriately.

150 Further extraction was performed, by adding $10 \mu 1$ of $1 \%$ TFA to the gel pieces, incubated for 5

151 mins at room temperature and shaking at $300 \mathrm{rpm}$. The extracted solution was added to the

152 digestion mixture. Then, $50 \mu \mathrm{L} \mathrm{ACN}$ was added to the gel pieces and incubated for 5 mins at

153 room temperature with $300 \mathrm{rpm}$ shaking, and the solution was removed and added to the

154 digestion mixture again. After that, $0.1 \%$ TFA was added to the gel pieces, incubated for 5 mins 
155 with $300 \mathrm{rpm}$ shaking at room temperature and repeated twice. All the supernatants were

156 combined, and speed vacuumed accordingly.

\section{LC-ESI-MS/MS analysis}

158 Before the sample loading, samples from three replicates of each method were reconstituted with $15930 \mu \mathrm{L}$ Solvent A and centrifuged at maximum speed for $10 \mathrm{mins}$. Spatial discrimination of the 160 peptide mixtures was achieved by loading $5 \mu \mathrm{L}$ of the digested peptides and packed into a large 161 capacity chip, 300A, C18, 160nL enrichment (Agilent) column and 75um x 150mm analytical

162 column (Agilent) with solvent A consisting of water with $0.1 \%$ formic acid and Solvent B 163 composed of $90 \%$ ACN in water with $0.1 \%$ formic acid. The gradient pump eluted with $20-80 \%$ $164 \mathrm{ACN}$ for $47 \mathrm{mins}$ and at a flow rate of $4 \mu \mathrm{L} / \mathrm{min}$ using Agilent 1200 series capillary pump and $1650.5 \mu \mathrm{L} / \mathrm{min}$ Nano pump coupled with Agilent $6550 \mathrm{iFunnel} \mathrm{Q-TOF} \mathrm{LC/MS/MS.} \mathrm{The} \mathrm{MS}$ 166 parameters used include positive ion polarity, 1900V capillary voltage with fragmentor voltage 167 of $360 \mathrm{~V}, 325^{\circ} \mathrm{C}$ gas temperature and $5.0 \mathrm{~L} / \mathrm{min}$ drying glass flow. Mass spectra from each spot in 168 the $\mathrm{m} / \mathrm{z}$ range obtained from 110 to $3000 \mathrm{~m} / \mathrm{z}$, whereby up to 500 laser shots accumulated per 169 spectra. The signal-to-noise $(\mathrm{S} / \mathrm{N})$ ratio set to a minimum of 10 , and the spots with the highest 170 intensity of precursor ion subjected to MS/MS analysis. A maximum of ten precursors was 171 allowed for the MS/MS analysis. For each spectrum, up to 2000 laser shots were accumulated 172 per spectrum, and the $\mathrm{S} / \mathrm{N}$ set to a minimum ratio of 15 . PeaksX was used to examine the MS 173 data against Uniprot Mosquito released 2020_01 from Swissprot and TrEMBL databases with a

174 fixed modification on Carbamidomethylation. A quantitative data normalization automatically 175 performed to correct any experimental bias, and the software calibrated to detect protein 176 threshold to $<1 \%$ FDR (false discovery rate). From the mass spectrometry data, proteins that 
177 showed scores $-10 \lg \mathrm{P} \geq 20 \quad(-(10 \log 10$ (P-value) $)$ and unique peptides $\geq 1$ in all replicates were used

178 for the subsequent analysis.

179

180 Functional annotation analysis

181 The list of all total proteins from each method obtained from the above analysis was assigned

182 functional categories using the Panther Classification System at www.pantherdb.org.

183

184

\section{Results}

185 Analysis of the total protein yield from three biological replicates showed that TCA acetone

186 precipitation extraction and CytoBuster ${ }^{\mathrm{TM}}$ reagent yielded means $283.15 \mu \mathrm{g}$ and $475.90 \mu \mathrm{g}$ of 187 proteins, respectively (Table 1).

189 The protein separation profile by $10 \%$ SDS-PAGE revealed the protein bands patterns in female 190 Ae. aegypti protein extracted with CytoBuster ${ }^{\mathrm{TM}}$ reagent and TCA acetone precipitation method 191 (Fig.1). There was dissimilarity pattern of protein bands between $250 \mathrm{kDa}-100 \mathrm{kDa}$ indicated 192 from both methods. However, more intense protein bands were observed in protein extracted 193 using CytoBuster ${ }^{\mathrm{TM}}$ reagent between $100 \mathrm{kDa}-10 \mathrm{kDa}$ though the bands pattern were similar in

194 both methods, as shown in Fig. 1.In the TCA acetone precipitation method and the CytoBuster ${ }^{\mathrm{TM}}$ 195 reagent protein extracts, a total of 890 and 1290 proteins were identified by LC-ESI-MS/MS, 196 respectively (Fig. 2 A and B). Analysis of LC-ESI-MS/MS of the CytoBuster ${ }^{\mathrm{TM}}$ reagent extract 197 showed the highest number of identified proteins than the TCA acetone precipitation method by 198 Wang et al. A total of 1797 proteins were identified by combining both methods, as shown in 199 Fig. 3. Meanwhile, Fig. 4 shows the combination of proteins identified in three replicates from 
200 each extraction method. LC-ESI-MS/MS_data of this study is available at proteomeXchange with 201 identifier number PXD019698.

202

203 There were eight categories of protein class from the proteins identified by LC-ESI-MS/MS

204 extracted using TCA acetone extraction method. The most abundant protein class was the 205 metabolite interconversion-enzyme class (PC00262), with 34.4\% hit (11 proteins) (Fig. 5A). The 206 least abundant protein classes were calcium-binding protein class (PC00060), chaperone protein 207 class (PC00072), and nucleic acid-binding protein class (PC00171) with 3.1\% hit (1 protein) in 208 each category, respectively (Fig. 5A). There were 11 protein classes from the proteins identified 209 using CytoBuster ${ }^{\mathrm{TM}}$. The most abundant protein class was also belonging to the metabolite 210 interconversion-enzyme protein class (PC00262) with 33.3\% hit (15 proteins) (Fig. 5B). The 211 least abundant classes were chaperone protein class (PC00072), protein-binding activity 212 modulator protein class (PC00095), nucleic acid-binding protein class (PC00171), a calcium213 binding protein class (PC00060), intercellular signal molecule protein class (PC00207) and 214 transporter protein class (PC00227) with 2.2\% hit (1 protein) each (Fig. 5B).

\section{Discussion}

217 This study evaluated two protein extraction methods to analyze female Ae. aegypti proteome 218 using LC-ESI-MS/MS. Among the two extraction methods, CytoBuster ${ }^{\mathrm{TM}}$ extraction reagent was 219 the fastest extraction method with high protein yield (Table 1). In contrast to this evaluation, 220 Cilia et al. reported that TCA acetone precipitation extraction method performed on Aphid 221 produced high protein yield of $20.4 \mathrm{mg} / \mathrm{g}$ when compared to phenol and multi-detergent 222 extraction methods yielded $7.3 \mathrm{mg} / \mathrm{g}$ and $4.79 \mathrm{mg} / \mathrm{g}$ protein, respectively (Cilia et al., 2009). In 
223 addition, protein identification analysis showed 188, 180 and 143 identified proteins from

224 extracts by TCA acetone precipitation, phenol and multi-detergent methods). Similarly, Hassan 225 et al. performed Plutella xylostella protein extraction using five different protocols. These 226 included TCA acetone with $0.7 \%$ 2-mercaptoethanol, TCA acetone with 2\% 2-mercaptoethanol, 227 TCA acetone with $40 \mathrm{mM}$ DTT, lysis buffer (7M urea, 2M thiourea, 4\% CHAPS) Tris HCL 228 method and PBS method. They concluded that TCA acetone with $40 \mathrm{mM}$ DTT yielded high total 229 protein amount of $25.17 \mathrm{mg} / \mathrm{g}$ with the most abundant protein spots of about 683 (Hassan et al., 230 2018). The TCA/acetone base extraction protocol is one of the most reported protein extraction 231 methods from various samples, including insects. TCA acetone protein extraction method has 232 continued to be an effective method in reducing protein degradation and get rid of interfering 233 elements (Hassan et al., 2018).

234

235 Visual observation of distinctive protein bands with no smearing showed good quality of protein 236 extract accomplished using both methods in this study (Fig. 1). Hence, the extracts were suitable 237 to be used for the subsequent proteomics analysis. Furthermore, the proteins extracted from 238 CytoBuster ${ }^{\mathrm{TM}}$ reagent were recommended, for protein separation using the two-dimensional 239 electrophoresis (2-DE) and Western blotting based on SDS-PAGE analysis where more intense 240 protein bands were obtained.

241

242 This study reported the first finding on the comparison of mosquito protein extraction methods 243 between a commercial reagent and TCA acetone precipitation method. The major drawback 244 attributed to the TCA acetone precipitation extraction method was the protein loss due to protein 245 precipitation and resoluble phases required in the process, coupled with many washing steps 
246 involved in the technique (Wu et al., 2014). At the same time, CytoBuster ${ }^{\mathrm{TM}}$ reagent allows the

247 isolation of active functional proteins without the need for additional washing and saves time.

248

249 The TCA acetone precipitation method and CytoBuster ${ }^{\mathrm{TM}}$ reagent gave 890 and 1290 proteins

250 identified by LC-ESI-MS/MS, respectively (Fig. 1 A and B). CytoBuster ${ }^{\mathrm{TM}}$ reagent showed the

251 highest number of identified proteins by LC-ESI-MS/MS than the TCA acetone precipitation.

252 The identified proteins from TCA acetone precipitation method and CytoBuster ${ }^{\mathrm{TM}}$ reagent

253 represent $5.34 \%$, and $7.75 \%$ of the total predicted Ae. aegypti proteins, respectively (Nene et al.,

254 2007; Morgat et al., 2020).

255

256 Furthermore, the total proteins identified by LC-ESI-MS/MS covered $10.3 \%$ Ae. aegypti

257 proteome by the combination of both methods in this study. Overall, we retrieved 29 well-

258 annotated/reviewed, 181 putative, 161 uncharacterized, and 1,398 hypotheticals proteins in this

259 study. Comparatively, Nunes et al. reported a total of 1139 identified proteins from female $A e$.

260 aegypti heads fed with blood and nectar exclusively, that induced differential protein expression

261 and the identified proteins stood at $7.4 \%$ of $A e$. aegypti proteins (Nunes et al., 2016). The

262 enriched number of identified proteins by Nunes et al. was due to off gel separation before LC-

263 MS/MS. There were 402 common proteins identified by LC-ESI-MS/MS in both extraction

264 methods (Fig. 3). The CytoBuster ${ }^{\mathrm{TM}}$ reagent had the highest number of 898 unique identified

265 proteins, suggesting a better coverage than TCA acetone precipitation method. A list of 20 top

266 proteins using LC-ESI-MS/MS can be found in supplementary data (Supplementary file 1).

267 On the other hand, analyses of proteins presence in three replicates from two extraction methods

268 (Fig.4), suggested by combining both extraction methods could represent a better protein 
269 identification and improved proteome coverage. This was shown by the lower number of

270 common identified proteins compared to the unique proteins of the both extraction methods.

271

272 There were eleven protein classes from identified proteins extracted by the CytoBuster ${ }^{\mathrm{TM}}$ reagent 273 compared to eight protein classes by TCA acetone precipitation extraction. We also identified 45 274 proteins and 11 protein class in CytoBuster ${ }^{\mathrm{TM}}$ in contrast to 32 proteins and 8 protein class in 275 TCA acetone precipitation method. Proteins associated with scaffold/adaptor protein (PC00226), 276 protein binding activity modulator (PC00095) and intercellular signal molecule (PC00207) were 277 only present in proteins extracted using CytoBuster ${ }^{\mathrm{TM}}$ reagent (Fig. 5A and B).

278

279 Among the unique proteins revealed by $\mathrm{CytoBuster}^{\mathrm{TM}}$ reagent is a 14-3-3 protein $\varepsilon$ 280 (Q7PX08/Q7PX08_ANOGA) that belongs to Scaffold/adaptor proteins. The 14-3-3- proteins

281 function as adapters, activators and repressors regulating signaling pathways in a range of 282 processes such as cell signaling. In Drosophila melanogaster, this protein interacts with many 283 regulators of the actin cytoskeleton (Ulvila et al., 2011). 14-3-3 protein $\varepsilon$ was crucial for 284 bacterial phagocytosis in Ae. aegypti and Ae. albopictus (Trujillo-Ocampo et al., 2017. Serine 285 proteases inhibitor (serpin) AGAP005246-PA (Q8WSX7/Q8WSX7_ANOGA) was also unique to CytoBuster ${ }^{\mathrm{TM}}$ reagent extracted proteins. They are acute phase response molecules and 287 regulate immune pathways for human pathogen transmission, and they belong to protein binding activity modulator (Gulley et al., 2013).

\section{Conclusion}

292 We performed a comparative analysis of two different protein extraction methods on female $A e$.

293 aegypti. CytoBuster ${ }^{\mathrm{TM}}$ reagent displayed a superior performance for the extraction of proteins in 
294

295

296

297

298

299

300

301

302

303

304

305

306

307

308

309

310

311

312

313

314

315

316

317

318

319

320

321

322

323

324

325

326

327

328

329

terms of protein yield, the proteome coverage identified by LC-ESI-MS/MS and extraction

speed. The functional analysis also revealed more proteins, and functions attributed to protein

extracted using the above reagent based on the rotein class. Nevertheless, combination of two

extraction methods could improve the proteome coverage in the presence study..

\section{References}

Cancino-Rodezno, A., Lozano, L., Oppert, C., Castro, J. I., Lanz-Mendoza, H., Encarnación, S., Evans, A. E., Gill, S. S., Soberón, M. and Jurat-Fuentes, J. L. 2012. Comparative proteomic analysis of Aedes aegypti larval midgut after intoxication with Cry11Aa toxin from Bacillus thuringiensis, PloS one, 7(5), pp. e37034.

Cilia, M., Fish, T., Yang, X., Mclaughlin, M., Thannhauser, T. and Gray, S. 2009. A comparison of protein extraction methods suitable for gel-based proteomic studies of aphid proteins, Journal of biomolecular techniques: JBT, 20(4), pp. 201.

Djegbe, I., Cornelie, S., Rossignol, M., Demettre, E., Seveno, M., Remoue, F. and Corbel, V. 2011. Differential expression of salivary proteins between susceptible and insecticideresistant mosquitoes of Culex quinquefasciatus, PLoS One, 6(3), pp. e17496.

Gulley, M. M., Zhang, X. and Michel, K. 2013. The roles of serpins in mosquito immunology and physiology, Journal of insect physiology, 59(2), pp. 138-147.

Hassan, M., YUSOFF, N., Aizat, W. M., Othman, N. W. and Abd Ghani, I. 2018.

Optimization Method for Proteomic Analysis of the Larva and Adult Tissues of Plutella xylostella (L.)(Lepidoptera: Plutellidae), Sains Malaysiana, 47(12), pp. 2975-2983.

Mano, C., Jariyapan, N., Sor-Suwan, S., Roytrakul, S., Kittisenachai, S., Tippawangkosol, P. and Somboon, P. 2019. Protein expression in female salivary glands of pyrethroidsusceptible and resistant strains of Aedes aegypti mosquitoes, Parasites \& vectors, 12(1), pp. 111.

Morgat, A., Lombardot, T., Coudert, E., Axelsen, K., Neto, T. B., Gehant, S., Bansal, P., Bolleman, J., Gasteiger, E. and De Castro, E. 2020. Enzyme annotation in UniProtKB using Rhea, Bioinformatics, 36(6), pp. 1896-1901.

Nene, V., Wortman, J. R., Lawson, D., Haas, B., Kodira, C., Tu, Z. J., Loftus, B., Xi, Z., Megy, K. and Grabherr, M. 2007. Genome sequence of Aedes aegypti, a major arbovirus vector, Science, 316(5832), pp. 1718-1723.

Nunes, A. T., Brito, N. F., Oliveira, D. S., Araujo, G. D., Nogueira, F. C. S., Domont, G. B., Moreira, M. F., Moreira, L. M., Soares, M. R. and Melo, A. C. 2016. Comparative proteome analysis reveals that blood and sugar meals induce differential protein expression in Aedes aegypti female heads, Proteomics, 16(19), pp. 2582-2586. 
Popova-Butler, A. and Dean, D. H. 2009. Proteomic analysis of the mosquito Aedes aegypti midgut brush border membrane vesicles, Journal of insect physiology, 55(3), pp. 264272.

Shashank, P. and Haritha, B. 2014. Insect proteomics: present and future prospective, Current Biotica, 7(4), pp. 336-342.

Trujillo-Ocampo, A., Cázares-Raga, F. E., del Angel, R. M., Medina-Ramírez, F., SantosArgumedo, L., Rodríguez, M. H. and de la Cruz Hernández-Hernández, F. 2017.

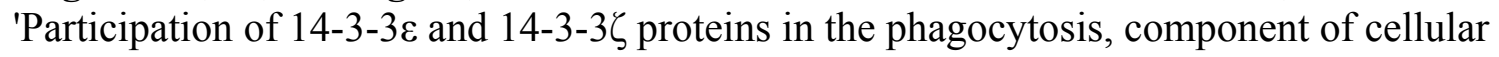
immune response, in Aedes mosquito cell lines, Parasites \& vectors, 10(1), pp. 1-12.

Ulvila, J., VANHA-AHO, L. M. and Rämet, M. 2011. Drosophila phagocytosis-still many unknowns under the surface', Apmis, 119(10), pp. 651-662.

Wang, W., Lv, Y., Fang, F., Hong, S., Guo, Q., Hu, S., Zou, F., Shi, L., Lei, Z. and Ma, K. 2015. Identification of proteins associated with pyrethroid resistance by iTRAQ-based quantitative proteomic analysis in Culex pipiens pallens, Parasites \& vectors, 8(1), pp. 111.

Whiten, S. R., Ray, W. K., Helm, R. F. and Adelman, Z. N. 2018. Characterization of the adult Aedes aegypti early midgut peritrophic matrix proteome using LC-MS, PloS one, 13(3), pp. e0194734.

Wu, X., Gong, F. and Wang, W. 2014. Protein extraction from plant tissues for $2 \mathrm{DE}$ and its application in proteomic analysis, Proteomics, 14(6), pp. 645-658. 


\section{Table 1 (on next page)}

Summary of protein extraction methods 
1

Table 1: Summary of protein extraction methods

TCA acetone precipitation method CytoBuster ${ }^{\mathrm{TM}}$ extraction reagent

Protein amount $(\mu \mathrm{g})$ mean $\pm \mathrm{SD} \quad 283.13 \mu \mathrm{g} \pm 255.49$

$475.87 \mu \mathrm{g} \pm 164.21$

No. of proteins identified

890

1290

Time

$50 \mathrm{hrs}$

$1 \mathrm{~h}$ 
Figure 1

Female Ae. aegypti proteins separated by 10\% SDS-PAGE

Lane 1 Protein ladder (Precision Plus Protein ${ }^{\text {TM }}$ Bio-Rad), Lane 2 Proteins extracted with TCA acetone precipitation method and Lane 3 Proteins extracted with CytoBuster ${ }^{\mathrm{TM}}$ reagent. 


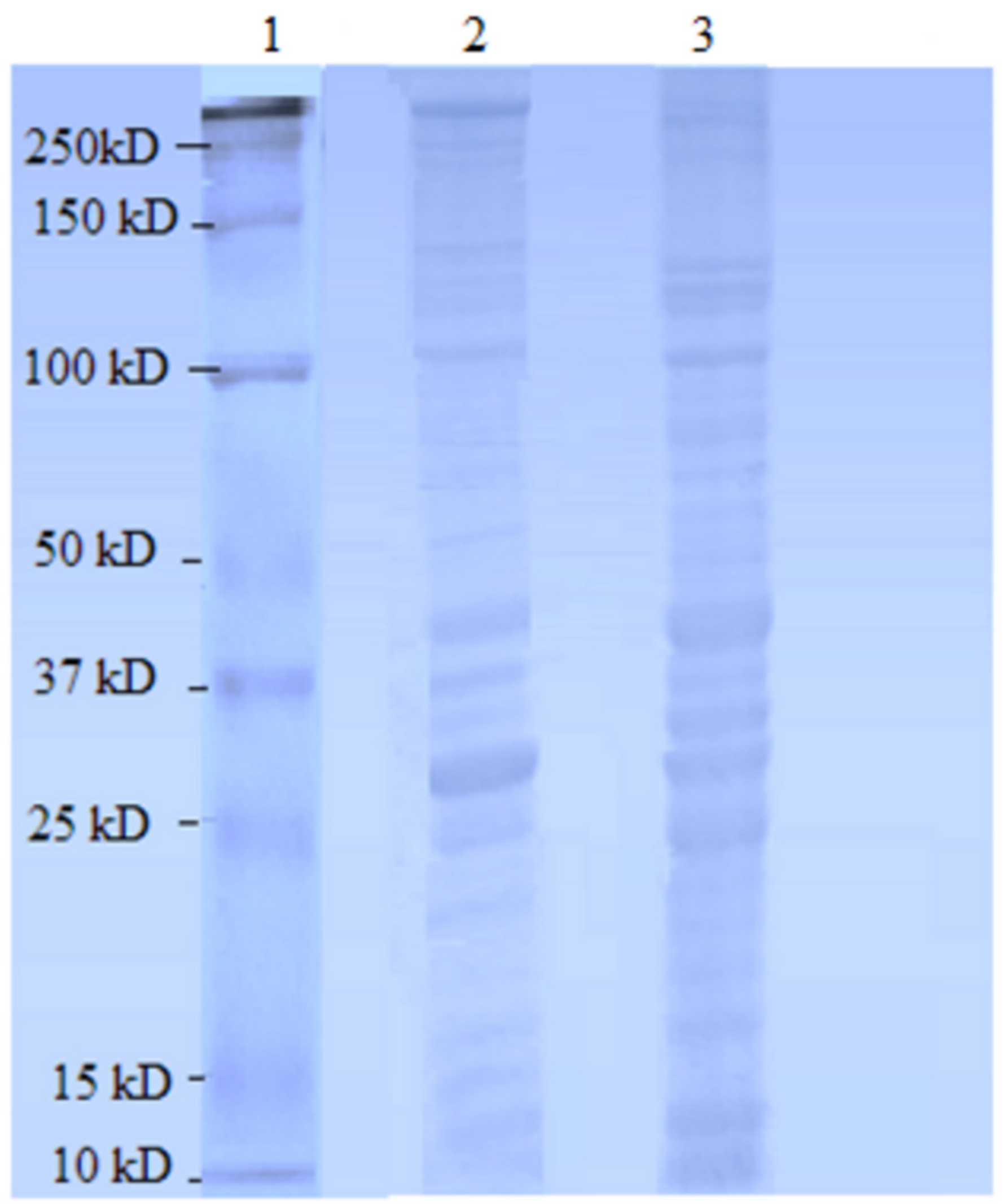




\section{Figure 2}

Number of identified proteins extracted using

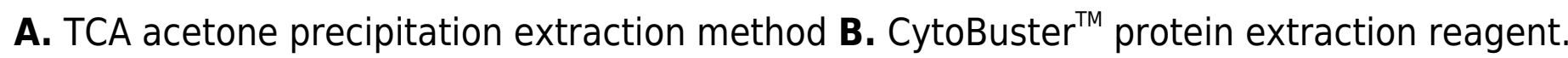
Ven diagrams were generated using BioVenn https://www. biovenn.nl/index.php .
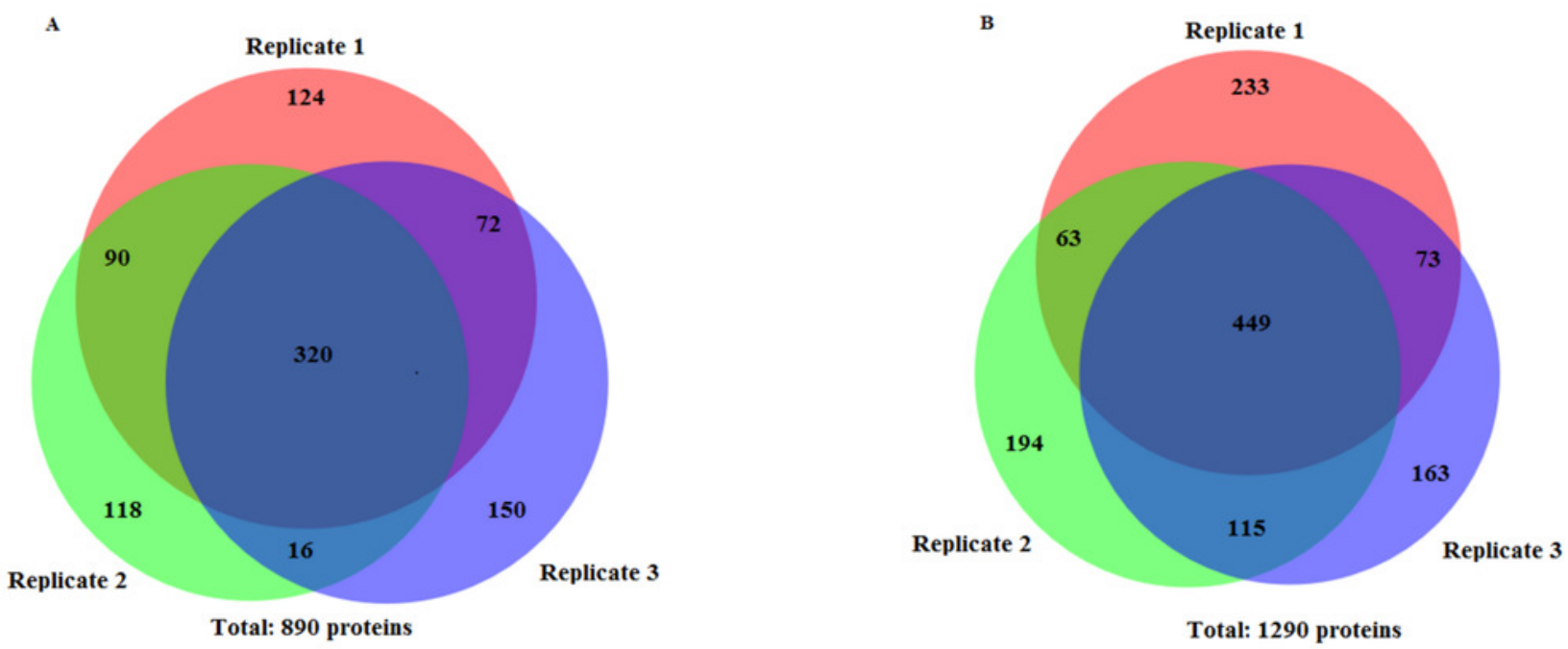
Figure 3

Unique and common proteins identified from TCA acetone precipitation and CytoBuster $^{\mathrm{TM}}$ extraction methods.

Ven diagram was generated BioVenn https://www.biovenn.nl/index.php . 
TCA acetone precipitation extraction method

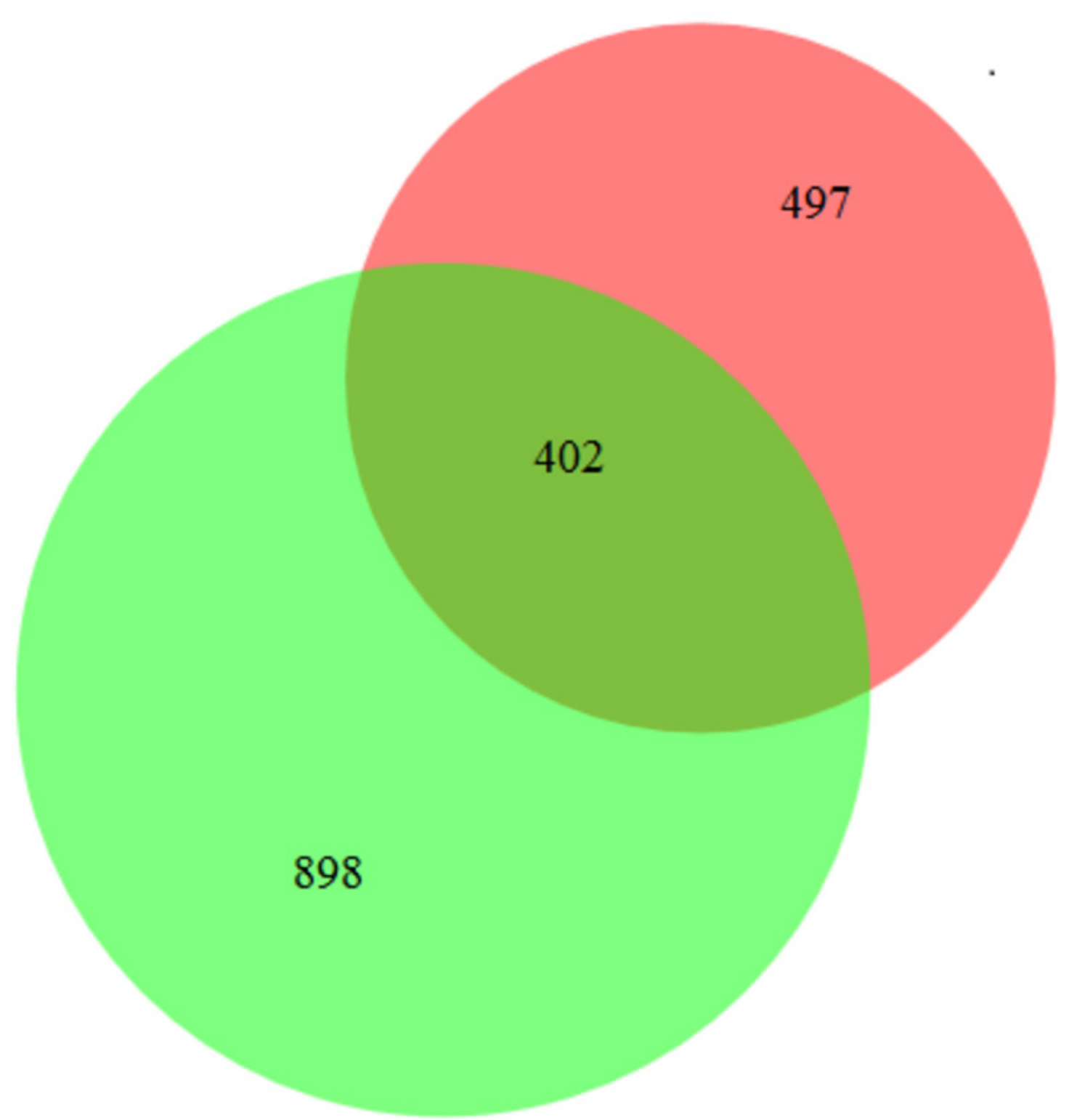

CytoBuster protein extraction reagent

Total: 1797 


\section{Figure 4}

Protein identified in three replicates.

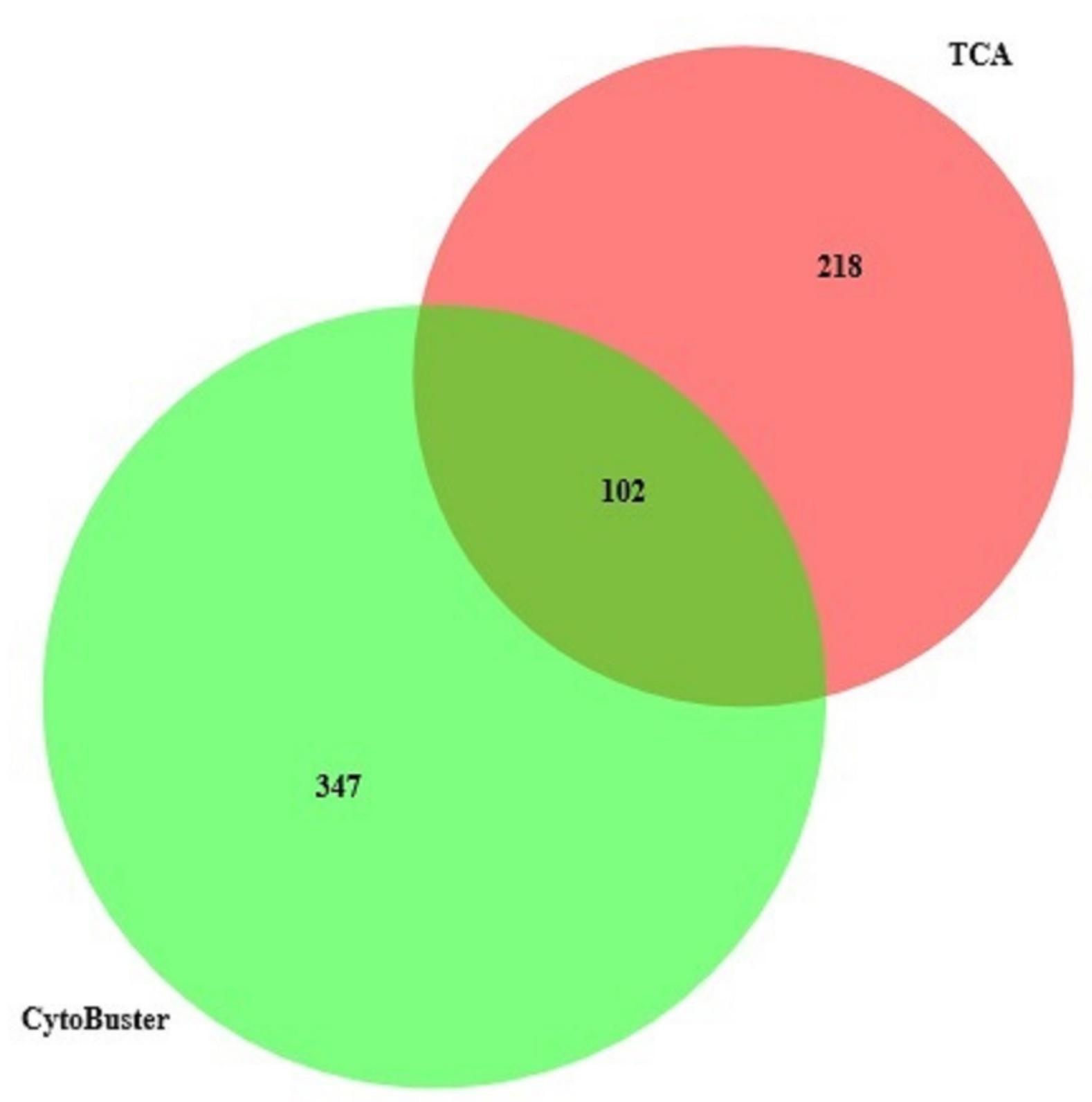




\section{Figure 5}

Protein Class categories using Panther version 15.0 released 2019-04.

A. TCA acetone precipitation extraction method. B. CytoBuster ${ }^{T M}$ protein extraction reagent.
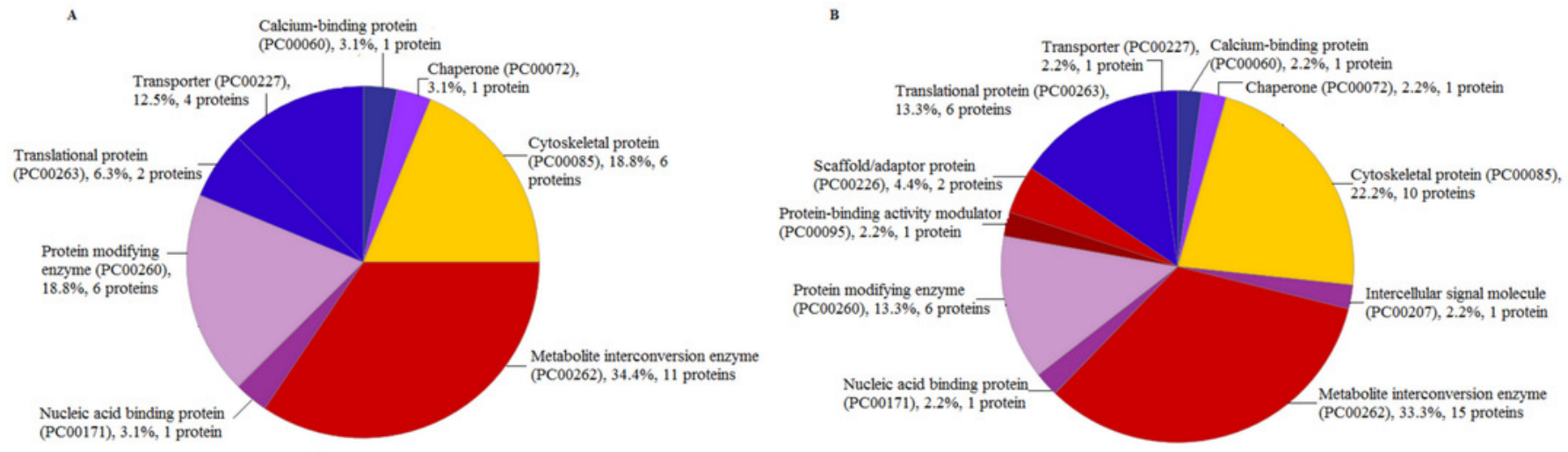